\title{
The 3-D extinction law in the 2nd quadrant of the Galactic disk
}

\section{Chao Liu ${ }^{1}$, Min Fang ${ }^{2}$, Yue $\mathrm{Wu}^{1}$, Kenneth Carrell ${ }^{1}$, Xiangxiang $\mathrm{Xue}^{1,3}$ and Glenn van de $\mathrm{Ven}^{3}$}

${ }^{1}$ Key Laboratory of Optical Astronomy, National Astronomical Observatories, CAS, 20A

Datun Road, Chaoyang District, 100012, Beijing, China

email: liuchao@nao.cas.cn

${ }^{2}$ Purple Mountain Observatory and Key Laboratory for Radio Astronomy, Chinese Acedamy of Sciences, 2 West Beijing Road, 210008, Nanjing, China

${ }^{3}$ Max Planck Institute for Astronomy, Königstuhl 17, D-69117 Heidelberg, Germany

\begin{abstract}
We estimate the 3D extinction law in the optical-NIR bands for a line of sight in the 2nd quadrant of the Galactic disk. Two dust lanes are identified at $\sim 9$ and $\sim 10.25 \mathrm{kpc}$ in both $A_{V}$ and $R_{V}$, indicating that the size of the dust grains in the spiral arms is larger than that in the inter-arm regions.
\end{abstract}

Keywords. dust, extinction, Galaxy: disk

\section{Introduction}

The interstellar extinction is related to the properties of the dust and varies with wavelength. The model of the extinction law can be parametrized with two parameter, $A_{V}$ and $R_{V}$ (Cardelli et al. (1989)). Both of the parameters are related to the distance as well as the direction of line of sight. Therefore, onlythe full 3D distribution can allow us to fully understand the extinction.

\section{Data and results}

We obtained 316 red clump star spectra observed from MMT/Hectospec at three 1-degree fields: $l=129^{\circ}$, $130^{\circ}$, and $131^{\circ}$ at $b=0^{\circ}$. The distances of the sample stars are measured according to Liu et al. (2012). Combining data from the SDSS and 2MASS catalogs, we

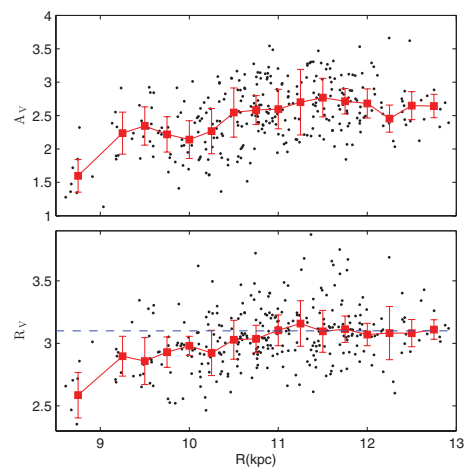

Figure 1. The estimated $A_{V}$ (top) and $R_{V}$ (bottom) as function of $R$ (dots). The red rectangles show the median values and the 1- $\sigma$ dispersions. estimate the reddening in 5 colors, i.e., $g-r, r-i, i-J, J-H$, and $H-K$, with accurate photometry. With these reddening values, we are able to estimate the extinction parameters, $A_{V}$ and $R_{V}$, as function of Galactocentric radius $R$ along this line of sight. Fig 1 shows the results. Two steeper rises are found at $\sim 9$ and $\sim 10.25 \mathrm{kpc}$, through which 2 dust lanes cross, in both $A_{V}$ and $R_{V}$. The correlation in $R_{V}$ makes it evident that the dust grain size in the spiral arms is larger than that in the inter-arm regions.

\section{References}

Cardelli, J. A., Clayton, G. C., \& Mathis, J. S. 1989, ApJ, 345, 245

Liu, C., Xue, X., Fang, M., van de Ven, G., Wu, Y., Smith, M., \& Carrell, K. 2012, ApJL, 753, 24 